\title{
Cocommutative Hopf Algebras of Permutations and Trees
}

\author{
MARCELO AGUIAR \\ maguiar@math.tamu.edu (http://www.math.tamu.edu/ maguiar) \\ FRANK SOTTILE \\ sottile@math.tamu.edu (http://www.math.tamu.edu/ sottile) \\ Department of Mathematics, Texas A\&M University, College Station, TX 77843, USA
}

Received March 12, 2004; Revised March 16, 2005; Accepted May 13, 2005

Abstract. Consider the coradical filtrations of the Hopf algebras of planar binary trees of Loday and Ronco and of permutations of Malvenuto and Reutenauer. We give explicit isomorphisms showing that the associated graded Hopf algebras are dual to the cocommutative Hopf algebras introduced in the late 1980's by Grossman and Larson. These Hopf algebras are constructed from ordered trees and heap-ordered trees, respectively. These results follow from the fact that whenever one starts from a Hopf algebra that is a cofree graded coalgebra, the associated graded Hopf algebra is a shuffle Hopf algebra.

Keywords: Hopf algebra, rooted tree, planar binary tree, symmetric group

\section{Introduction}

In the late 1980's, Grossman and Larson constructed several cocommutative Hopf algebras from different families of trees (rooted, ordered, heap-ordered), in connection to the symbolic algebra of differential operators $[10,11]$. Other Hopf algebras of trees have arisen lately in a variety of contexts, including the Connes-Kreimer Hopf algebra in renormalization theory [5] and the Loday-Ronco Hopf algebra in the theory of associativity breaking [18, 19]. The latter is closely related to other important Hopf algebras in algebraic combinatorics, including the Malvenuto-Reutenauer Hopf algebra [22] and the Hopf algebra of quasisymmetric functions [21,27,31].

This universe of Hopf algebras of trees is summarized below.

\begin{tabular}{lll}
\hline & Family of trees & \multicolumn{1}{c}{ Hopf algebra } \\
\hline Grossman-Larson [10, 11] & $\begin{array}{l}\text { Rooted trees } \\
\text { Ordered trees }\end{array}$ & Non-commutative, cocommutative \\
& Heap-ordered trees & \\
Loday-Ronco [18] & Planar binary trees & Non-commutative, non-cocommutative \\
Connes-Kreimer [5] & Rooted trees & Commutative, non-cocommutative \\
\hline
\end{tabular}

Aguiar supported in part by NSF grant DMS-0302423. Sottile supported in part by NSF CAREER grant DMS0134860, the Clay Mathematics Institute, and MSRI. 
Recent independent work of Foissy [6,7] and Hoffman [14] showed that the Hopf algebra of Connes-Kreimer is dual to the Hopf algebra of rooted trees of Grossman-Larson. This Hopf algebra also arises as the universal enveloping algebra of the free pre-Lie algebra on one generator, viewed as a Lie algebra [4]. Foissy [7] and Holtkamp [15] showed that the Hopf algebra of Connes-Kreimer is a quotient of the Hopf algebra of Loday-Ronco, see also [3].

We give explicit isomorphisms which show that the Grossman-Larson Hopf algebras of ordered trees and of heap-ordered trees are dual to the associated graded Hopf algebras to the Hopf algebra $\mathcal{Y}$ Sym of planar binary trees of Loday and Ronco and the Hopf algebra SSym of permutations of Malvenuto and Reutenauer, respectively. This is done in Theorems 2.5 and 3.4. The case of heap-ordered trees requires the assumption that the base field be of characteristic 0 . We establish this case in Section 3 by making use of the first Eulerian idempotent.

The essential tool we use is the monomial basis of Y Sym and SSym introduced in our previous works $[2,3]$. The explicit isomorphisms are in terms of the dual bases of ordered and heap-ordered trees of Grossman-Larson and of the monomial bases of $\mathcal{Y} S y m$ and SSym, respectively. These results provide unexpected combinatorial descriptions for the associated graded Hopf algebras to YSym and SSym. On the other hand, together with the result of Foissy and Hoffman, they connect all Grossman-Larson Hopf algebras of trees to the mainstream of combinatorial Hopf algebras.

It follows from our results that the associated graded Hopf algebras to the Hopf algebras of Loday-Ronco and Malvenuto-Reutenauer are commutative, a fact which is not obvious from the explicit description of the product of these algebras. Greg Warrington noticed this for the Malvenuto-Reutenauer Hopf algebra and Loïc Foissy made us aware that the associated graded Hopf algebra to any graded connected Hopf algebra is always commutative (private communications). A related well-known fact is that the associated graded Hopf algebra to a cofree graded connected Hopf algebra is a shuffle Hopf algebra. We recall these and related results with their proofs in Section 1. This also implies that the algebras of Grossman and Larson are tensor Hopf algebras (Corollaries 2.7 and 3.6). It was known from [10] that these algebras are free.

\section{Cofree graded coalgebras and Hopf algebras}

A coalgebra $(C, \Delta, \epsilon)$ over a field $\mathbb{k}$ is called graded if there is given a decomposition $C=\oplus_{k \geq 0} C^{k}$ of $C$ as a direct sum of $\mathbb{k}$-subspaces $C^{k}$ such that

$$
\Delta\left(C^{k}\right) \subseteq \sum_{i+j=k} C^{i} \otimes C^{j} \text { and } \epsilon\left(C^{k}\right)=0 \forall k \neq 0
$$

The coalgebra is said to be graded connected if in addition $C^{0} \cong \mathbb{k}$.

Definition 1.1 A graded coalgebra $Q=\oplus_{k \geq 0} Q^{k}$ is said to be cofree if it satisfies the following universal property. Given a graded coalgebra $C=\oplus_{k \geq 0} C^{k}$ and a linear map $\varphi: C \rightarrow Q^{1}$ with $\varphi\left(C^{k}\right)=0$ when $k \neq 1$, there is a unique morphism of graded coalgebras 
$\hat{\varphi}: C \rightarrow Q$ such that the following diagram commutes

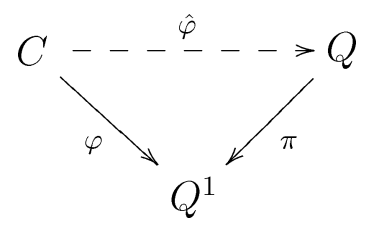

where $\pi: Q \rightarrow Q^{1}$ is the canonical projection.

Let $V$ be a vector space and set

$$
Q(V):=\bigoplus_{k \geq 0} V^{\otimes k}
$$

We write elementary tensors from $V^{\otimes k}$ as $x_{1} \backslash x_{2} \backslash \cdots \backslash x_{k}\left(x_{i} \in V\right)$ and identify $V^{\otimes 0}$ with $\mathbb{k}$. The space $Q(V)$, graded by $k$, becomes a graded connected coalgebra with the deconcatenation coproduct

$$
\Delta\left(x_{1} \backslash x_{2} \backslash \cdots \backslash x_{k}\right)=\sum_{i=0}^{k}\left(x_{1} \backslash \cdots \backslash x_{i}\right) \otimes\left(x_{i+1} \backslash \cdots \backslash x_{k}\right)
$$

and counit given by projection onto $V^{\otimes 0}=\mathbb{k}$. Moreover, $Q(V)$ is a cofree graded coalgebra [33, Lemma 12.2.7]. It is in fact graded connected.

By universality, any cofree graded coalgebra $Q$ is isomorphic to $Q(V)$, where $V=Q^{1}$. We refer to $Q(V)$ as the cofree graded coalgebra cogenerated by $V$.

Remark 1.3 The functor $Q$ from vector spaces to graded coalgebras is right adjoint to the forgetful functor $C \mapsto C^{1}$ from graded coalgebras to vector spaces. $Q(V)$ is not cofree in the category of all coalgebras over $\mathbb{k}$. However, $Q(V)$ is still cofree in the category of connected coalgebras in the sense of Quillen [26, Appendix B, Proposition 4.1]. See also [33, Theorem 12.0.2].

We are interested in Hopf algebra structures on cofree graded coalgebras. There is recent important work of Loday and Ronco in this direction [20], but their results are not prerequisites for our work.

In the classical Hopf algebra literature usually only one Hopf algebra structure on $Q(V)$ is considered: the shuffle Hopf algebra. It is well-known that this is the only Hopf algebra structure on $Q(V)$ for which the algebra structure preserves the grading; this may be deduced from [33, Theorem 12.1.4] but we provide a direct proof below (Proposition 1.4). There are, however, many naturally occurring Hopf algebras that are cofree graded coalgebras and for which the algebra structure does not preserve the grading; see Examples 1.8. 
The shuffle Hopf algebra. Let $V$ be an arbitrary vector space. There is an algebra structure on $Q(V)$ defined recursively by

$$
x \cdot 1=x=1 \cdot x
$$

for $x \in V$, and

$$
\begin{aligned}
\left(x_{1} \backslash \cdots \backslash x_{j}\right) \cdot\left(y_{1} \backslash \cdots \backslash y_{k}\right)= & x_{1} \backslash\left(\left(x_{2} \backslash \cdots \backslash x_{j}\right) \cdot\left(y_{1} \backslash \cdots \backslash y_{k}\right)\right) \\
& +y_{1} \backslash\left(\left(x_{1} \backslash \cdots \backslash x_{j}\right) \cdot\left(y_{2} \backslash \cdots \backslash y_{k}\right)\right) .
\end{aligned}
$$

Together with the graded coalgebra structure (1.2), this gives a Hopf algebra which is denoted $\operatorname{Sh}(V)$ and called the shuffle Hopf algebra of $V$.

A Hopf algebra $H$ is called graded if it is a graded coalgebra and the multiplication and unit preserve the grading:

$$
H^{j} \cdot H^{k} \subseteq H^{j+k}, \quad 1 \in H^{0} .
$$

The shuffle Hopf algebra $\operatorname{Sh}(V)$ is a graded Hopf algebra. As mentioned, it is the only such structure that a cofree graded coalgebra admits.

Proposition 1.4 Let $H=\oplus_{k \geq 0} H^{k}$ be a graded Hopf algebra which is cofree as a graded coalgebra. Then there is an isomorphism of graded Hopf algebras

$$
H \cong \operatorname{Sh}\left(H^{1}\right) .
$$

Proof: We may assume that $H=Q(V)$, with $V=H^{1}$. By hypothesis, the multiplication map is a morphism of graded Hopf algebras $m: H \otimes H \rightarrow H$, where the component of degree $k$ of $H \otimes H$ is $\sum_{i+j=k} H^{i} \otimes H^{j}$. By cofreeness, $m$ is uniquely determined by the composite

$$
H \otimes H \stackrel{m}{\rightarrow} H \stackrel{\pi}{\rightarrow} H^{1},
$$

which in turn reduces to

$$
(H \otimes H)^{1}=H^{0} \otimes H^{1}+H^{1} \otimes H^{0} \stackrel{m}{\rightarrow} H^{1} .
$$

Also by hypothesis, $H^{0}=\mathbb{k} \cdot 1$ where 1 is the unit element of $H$. Hence the above map, and then $m$, are determined by

$$
1 \otimes x \mapsto x \text { and } x \otimes 1 \mapsto x
$$

This shows that there is a unique multiplication on $H$ that makes it a graded Hopf algebra. Since the multiplication of the shuffle Hopf algebra of $H^{1}$ is one such map, it is the only one. Thus, $H$ is the shuffle Hopf algebra of $H^{1}$. 
The tensor Hopf algebra. Let $V$ be a vector space and set

$$
T(V):=\bigoplus_{k \geq 0} V^{\otimes k}
$$

As a vector space, $T(V)=Q(V)$. The space $T(V)$ becomes a graded algebra under the concatenation product

$$
\left(x_{1} \backslash \cdots \backslash x_{i}\right) \cdot\left(y_{1} \backslash \cdots \backslash y_{j}\right)=x_{1} \backslash \cdots \backslash x_{i} \backslash y_{1} \backslash \cdots \backslash y_{j}
$$

and unit $1 \in V^{\otimes 0}=\mathbb{k}$. Moreover, $T(V)$ is the free algebra on $V$.

If $V$ is finite dimensional, the graded dual of $Q(V)$ is the tensor algebra $T\left(V^{*}\right)$.

There is a graded Hopf algebra structure on $T(V)$ uniquely determined by

$$
\Delta(x)=1 \otimes x+x \otimes 1 \quad \text { and } \quad \epsilon(x)=0
$$

for $x \in V$. This is the tensor Hopf algebra. An argument dual to that of Proposition 1.4 shows that it is the only graded Hopf algebra structure that a free algebra admits.

The coradical filtration. Let $H=\oplus_{k \geq 0} H^{k}$ be a Hopf algebra that is graded as a coalgebra. We do not insist that the algebra structure of $H$ preserves this grading. Let $F^{0}(H):=H^{0}$ and let $F^{k}(H)$ consist of those elements $h \in H$ such that in the iterated coproduct $\Delta^{(k)}(h)$ every term has a tensor factor from $F^{0}(H)$. It follows that $F^{k}(H) \subseteq F^{k+1}(H)$ and $H^{k} \subseteq F^{k}(H)$.

Suppose $H$ is connected, i.e., $F^{0}(H)=H^{0}=\mathbb{k}$. In this case, $F^{0}(H)$ is the coradical of $H$ and the subspaces $F^{k}(H)$ form the coradical filtration of $H$ [24, Chapter 5]. It is known that

$$
\begin{aligned}
& H=\bigcup_{k \geq 0} F^{k}(H), \quad \Delta\left(F^{k}(H)\right) \subseteq \sum_{i+j=k} F^{i}(H) \otimes F^{j}(H), \text { and } \\
& F^{j}(H) \cdot F^{k}(H) \subseteq F^{j+k}(H) .
\end{aligned}
$$

These results hold in greater generality; see [24, Theorem 5.2.2, Lemma 5.2.8].

Let $\operatorname{gr}(H)$ be the graded Hopf algebra associated to the coradical filtration,

$$
\operatorname{gr}(H)=F^{0}(H) \oplus F^{1}(H) / F^{0}(H) \oplus \cdots \oplus F^{k+1}(H) / F^{k}(H) \oplus \cdots
$$

If $m$ and $\Delta$ are the operations of $H$, then the operations of $\operatorname{gr}(H)$ are induced by the compositions

$$
\begin{aligned}
& F^{j}(H) \otimes F^{k}(H) \stackrel{m}{\longrightarrow} F^{j+k}(H) \longrightarrow F^{j+k}(H) / F^{j+k-1}(H), \\
& \quad F^{k}(H) \stackrel{\Delta}{\longrightarrow} \sum_{i+j=k} F^{i}(H) \otimes F^{j}(H) \longrightarrow \sum_{i+j=k} F^{i}(H) / F^{i-1}(H) \otimes F^{j}(H) / F^{j-1}(H) .
\end{aligned}
$$

The main goal of this paper is to obtain explicit combinatorial descriptions for the associated graded Hopf algebras to the Hopf algebras YSym and SSym of Examples 1.8. This is done 
in Sections 2 and 3. These Hopf algebras are cofree graded coalgebras, so we discuss the coradical filtration for such Hopf algebras first.

Let $H=Q(V)$ be a Hopf algebra that is a cofree graded coalgebra. We have $H^{0}=\mathbb{k}$, $H^{1}=V=P(H)$, the space of primitive elements of $H$, and $H^{k}=V^{\otimes k}$. As before, we do not require that the algebra structure of $H$ preserves this grading. It is easy to see that

$$
F^{k}(H):=H^{0} \oplus H^{1} \oplus \cdots \oplus H^{k} .
$$

Therefore, $\operatorname{gr}(H) \cong H$ as graded coalgebras canonically, and the multiplication has been altered by removing terms of lower degree from a homogeneous product. More precisely, if $m$ is the multiplication map on $H$, then the multiplication on $\operatorname{gr}(H)$ is the composition

$$
H^{j} \otimes H^{k} \stackrel{m}{\longrightarrow} F^{j+k}(H) \longrightarrow H^{j+k}
$$

Proposition 1.5 Let $H$ be a Hopf algebra that is a cofree graded coalgebra. Then its associated graded Hopf algebra $\operatorname{gr}(H)$ is the shuffle Hopf algebra $\operatorname{Sh}\left(H^{1}\right)$. In particular, $\operatorname{gr}(H)$ is commutative.

Proof: Since $H \cong \operatorname{gr}(H)$ as graded coalgebras, Proposition 1.4 applies to $\operatorname{gr}(H)$.

The commutativity of the associated graded Hopf algebra holds in greater generality. The following result was pointed out to us by Foissy.

Proposition 1.6 Let $H$ be a graded connected Hopf algebra. Then $\operatorname{gr}(H)$ is commutative.

Proof: We show that $\left[F^{j}(H), F^{k}(H)\right] \subseteq F^{j+k-1}(H)$, and hence commutators vanish in $\operatorname{gr}(H)$.

It follows from the definition of the coradical filtration that for any $h \in F^{a}(H)$ every term in $\Delta^{(a+b-1)}(h)$ contains at least $b$ factors from $F^{0}(H)=\mathbb{k}$.

Let $x \in F^{j}(H)$ and $y \in F^{k}(H)$. Every term in $\Delta^{(j+k-1)}(x)$ contains at least $k$ factors from $\mathbb{k}$ and every term in $\Delta^{(j+k-1)}(y)$ contains at least $j$ factors from $\mathbb{k}$. Write

$$
\Delta^{(j+k-1)}(x)=\sum x_{1} \otimes \cdots \otimes x_{j+k} \quad \text { and } \quad \Delta^{(j+k-1)}(y)=\sum y_{1} \otimes \cdots \otimes y_{j+k} .
$$

Consider those terms in

$$
\Delta^{(j+k-1)}(x y)=\Delta^{(j+k-1)}(x) \Delta^{(j+k-1)}(y)=\sum x_{1} y_{1} \otimes \cdots \otimes x_{j+k} y_{j+k}
$$

in which none of the $j+k$ factors are from $\mathbb{k}$. By the pigeon-hole principle, these terms must be such that for each $i=1, \ldots, j+k$ either $x_{i} \in \mathbb{k}$ or $y_{i} \in \mathbb{k}$. Therefore, these terms satisfy

$$
x_{1} y_{1} \otimes \cdots \otimes x_{j+k} y_{j+k}=y_{1} x_{1} \otimes \cdots \otimes y_{j+k} x_{j+k} .
$$


The right-hand side is a term in $\Delta^{(j+k-1)}(y x)$, and by symmetry this gives all terms in $\Delta^{(j+k-1)}(y x)$ in which none of the factors are from $\mathbb{k}$. These cancel in $\Delta^{(j+k-1)}(x y-y x)$. Thus, every term in $\Delta^{(j+k-1)}(x y-y x)$ contains at least one factor from $\mathbb{k}$, which proves that $x y-y x \in F^{j+k-1}(H)$.

Remark 1.7 Consider the coradical filtration of an arbitrary (not necessarily graded or connected) Hopf algebra. The same argument as above shows that if the coradical $F^{0}(H)$ lies in the center of $H$, then the associated graded Hopf algebra is commutative.

The cofree graded coalgebras we are interested in carry a second grading. With respect to this second grading, but not with respect to the original one, they are in fact graded Hopf algebras. The general setup is as follows.

Suppose $V=\oplus_{i \geq 1} V_{i}$ is a graded space and each $V_{i}$ is finite dimensional. Then $Q(V)$ carries another grading, for which the elements of $V_{i_{1}} \otimes \cdots \otimes V_{i_{k}}$ have degree $i_{1}+\cdots+i_{k}$. In this situation, we refer to $k$ as the length and to $i_{1}+\cdots+i_{k}$ as the weight. The homogeneous components of the two gradings on $Q(V)$ are thus

$$
Q(V)^{k}:=V^{\otimes k} \quad \text { and } \quad Q(V)_{n}:=\bigoplus_{\substack{k \geq 0 \\ i_{1}+\cdots+i_{k}=n}} V_{i_{1}} \otimes \cdots \otimes V_{i_{k}} .
$$

Note that each $Q(V)_{n}$ is finite dimensional. Let $V^{*}:=\oplus_{i \geq 1} V_{i}^{*}$ denote the graded dual of $V$. The graded dual of $Q(V)$ with respect to the grading by weight is the tensor algebra $T\left(V^{*}\right)$, and the graded dual of $\operatorname{Sh}(V)$ with respect to the grading by weight is the tensor Hopf algebra $T\left(V^{*}\right)$.

Example 1.8 We give some examples of cofree graded coalgebras.

(1) The Hopf algebra of quasi-symmetric functions. This Hopf algebra, often denoted $\mathcal{Q S y m}$, has a linear basis $M_{\alpha}$ indexed by compositions $\alpha=\left(a_{1}, \ldots, a_{k}\right)$ (sequences of positive integers). See $[21,27,31]$ for more details. $\mathcal{Q}$ Sym is a cofree graded coalgebra, as follows. Let $V$ be the subspace linearly spanned by the elements $M_{(n)}, n \geq 1$. Then $\mathcal{Q S y m} \cong Q(V)$ via

$$
M_{\left(a_{1}, \ldots, a_{k}\right)} \leftrightarrow M_{\left(a_{1}\right)} \backslash \cdots \backslash M_{\left(a_{k}\right)} .
$$

This isomorphism identifies $V^{\otimes k}$ with the subspace of $\mathcal{Q} S y m$ spanned by the elements $M_{\alpha}$ indexed by compositions of length $k$. QSym is not a shuffle Hopf algebra: the product does not preserve the grading by length. For instance,

$$
M_{(n)} \cdot M_{(m)}=M_{(n, m)}+M_{(m, n)}+M_{(n+m)} .
$$

In this case, $V$ is graded by $n$, and the grading by weight assigns degree $a_{1}+\cdots+a_{k}$ to $M_{\alpha}$. The Hopf algebra structure of $\mathcal{Q}$ Sym does preserve the grading by weight. 
This is an example of a quasi-shuffle Hopf algebra [12,13]. According to [13, Theorem 3.3], any (commutative) quasi-shuffle Hopf algebra is isomorphic to a shuffle Hopf algebra. The isomorphism does not however preserve the grading by length, and thus its structure as a cofree graded coalgebra. For more on the cofreeness of QSym, see [1, Theorem 4.1].

(2) The Hopf algebra of planar binary trees. This Hopf algebra was introduced by Loday and Ronco $[18,19]$. We denote it by $\mathcal{Y} S y m$. It is known that $\mathcal{Y}$ Sym is a cofree graded coalgebra [3, Theorem 7.1, Corollary 7.2]. The product of $\mathcal{Y} S y m$ does not preserve the grading by length (but it preserves the grading by weight). $\mathcal{Y}$ Sym is not a shuffle Hopf algebra, not even a quasi-shuffle Hopf algebra. See Section 2 for more details.

(3) The Hopf algebra of permutations. This Hopf algebra was introduced by Malvenuto and Reutenauer [21,22]. We denote it by $\mathfrak{S} S y m$. As for $\mathcal{Y} S y m, \mathfrak{S} S y m$ is a cofree graded coalgebra [2, Theorem 6.1, Corollary 6.3] and is neither a shuffle nor quasi-shuffle Hopf algebra. See Section 3 for more details.

(4) The Hopf algebra of peaks. This Hopf algebra was introduced by Stembridge [32] and is often denoted $\Pi$. It has a linear basis indexed by odd compositions (sequences of non-negative odd integers). It has been recently shown that $\Pi$ is a cofree graded coalgebra [16, Theorem 4.3], see also [29, Proposition 3.3].

\section{The Hopf algebra of ordered trees}

We show that the graded dual to $\operatorname{gr}(\mathcal{Y} S y m)$ is isomorphic to the cocommutative Hopf algebra of ordered trees defined by Grossman and Larson [10].

We first review the definition of the Hopf algebra of ordered trees.

For the definition of ordered trees (also called rooted planar trees), see [30, page 294]. The ordered trees with 1, 2, 3, and 4 nodes are shown below:

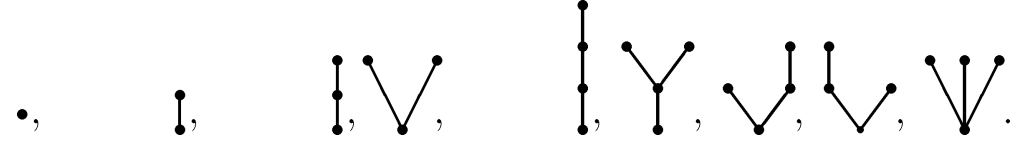

Given two ordered trees $x$ and $y$, we may join them together at their roots to obtain another ordered tree $x \backslash y$, where the nodes of $x$ are to the left of those of $y$ :

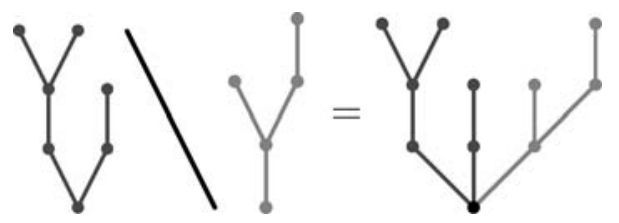

An ordered tree is planted if its root has a unique child. Every ordered tree $x$ has a unique decomposition

$$
x=x_{1} \backslash \cdots \backslash x_{k}
$$

into planted trees $x_{1}, \ldots, x_{k}$, corresponding to the branches at the root of $x$. These are the planted components of $x$. 
The set of nodes of an ordered tree $x$ is denoted by $\operatorname{Nod}(x)$. Let $x$ be an ordered tree and $x_{1}, \ldots, x_{k}$ its planted components, listed from left to right and (possibly) with multiplicities. Given a function $f:[k] \rightarrow \operatorname{Nod}(y)$ from the set $[k]=\{1, \ldots, k\}$ to the set of nodes of another ordered tree $y$, form a new ordered tree $x \#_{f} y$ by identifying the root of each component $x_{i}$ of $x$ with the corresponding node $f(i)$ of $y$. For this to be an ordered tree, retain the order of any components of $x$ attached to the same node of $y$, and place them to the left of any children of that node in $y$. Given a subset $S \subseteq[k]$, say $S=\left\{i_{1}<\cdots<i_{p}\right\}$, let

$$
x_{S}:=x_{i_{1}} \backslash \cdots \backslash x_{i_{p}}
$$

Equivalently, $x_{S}$ is the tree obtained by erasing the branches at the root of $x$ which are not indexed by $S$. Let $S^{c}=[k] \backslash S$.

Definition 2.2 The Grossman-Larson Hopf algebra $\mathcal{H}_{O}$ of ordered trees is the formal linear span of all ordered trees with product and coproduct as follows. Given ordered trees $x$ and $y$ as above, we set

$$
\begin{aligned}
& x \cdot y=\sum_{f:[k] \rightarrow \operatorname{Nod}(y)} x_{f} y, \\
& \Delta(x)=\sum_{S \subseteq[k]} x_{S} \otimes x_{S^{c}},
\end{aligned}
$$

the first sum is over all functions from $[k]$ to the set of nodes of $y$ and the second is over all subsets of $[k] . \mathcal{H}_{O}$ is a graded Hopf algebra, where the degree of an ordered tree is one less than the number of nodes [10, Theorem 3.2].

We give some examples, using colors to indicate how the operations are performed (they are not part of the structure of an ordered tree).

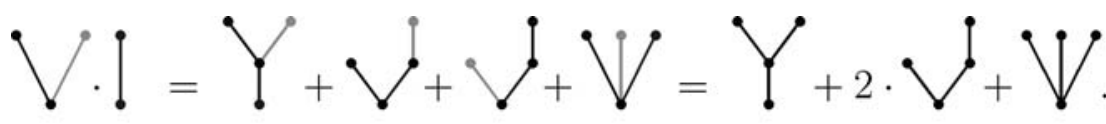

$$
\begin{aligned}
& \Delta(\bigvee)=\bullet \otimes+i \otimes+i \otimes V+i \otimes V
\end{aligned}
$$

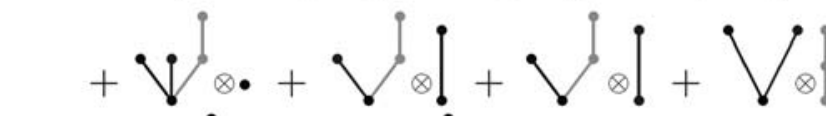

$$
\begin{aligned}
& =\bullet \otimes+2 \cdot i \otimes \downarrow+i \otimes V
\end{aligned}
$$

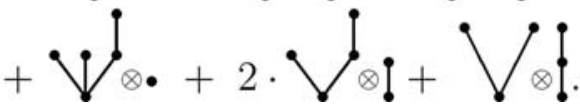


The definition implies that $\mathcal{H}_{O}$ is cocommutative and that each planted tree is a primitive element in $\mathcal{H}_{O}$. (There are other primitive elements. In fact, $\mathcal{H}_{O}$ is isomorphic to the tensor Hopf algebra on the subspace spanned by the set of planted trees. See Corollary 2.7.)

We follow the notation and terminology of [3] for planar binary trees and the LodayRonco Hopf algebra YSym (much of which is based on the constructions of $[18,19]$ ).

Ordered trees are in bijection with planar binary trees. Given a planar binary tree $t$, draw a node on each of its leaves, then collapse all edges of the form /. The resulting planar graph, rooted at the node coming from the right-most leaf of $t$, is an ordered tree. This defines a bijection $\psi$ from planar binary trees with $n$ leaves to ordered trees with $n$ nodes.

We will make use of a recursive definition of $\psi$. Recall the operation $s \backslash t$ between planar binary trees, which is obtained by identifying the right-most leaf of $s$ with the root of $t$ (putting $s$ under $t$ ). For instance,

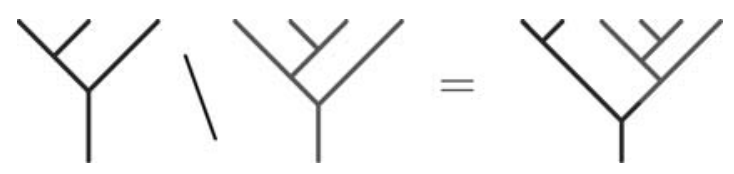

This operation is associative and so any planar binary tree $t$ has a unique maximal decomposition

$$
t=t_{1} \backslash t_{2} \backslash \cdots \backslash t_{k}
$$

in which each $t_{i}$ is $\backslash$-irreducible. Note that a planar binary tree $t$ is $\backslash$-irreducible precisely when it is of the form

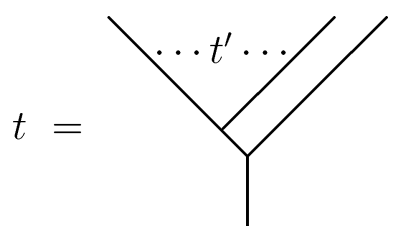

for some planar binary tree $t^{\prime}$ with one less leaf than $t$.

The bijection $\psi$ may be computed recursively as follows. First, for $t$ as in (2.3),

$$
\psi(t)=\psi\left(t_{1}\right) \backslash \psi\left(t_{2}\right) \backslash \cdots \backslash \psi\left(t_{k}\right) .
$$

Second, for $t$ as in (2.4), $\psi(t)$ is obtained by adding a new root to the ordered tree $\psi\left(t^{\prime}\right)$ :

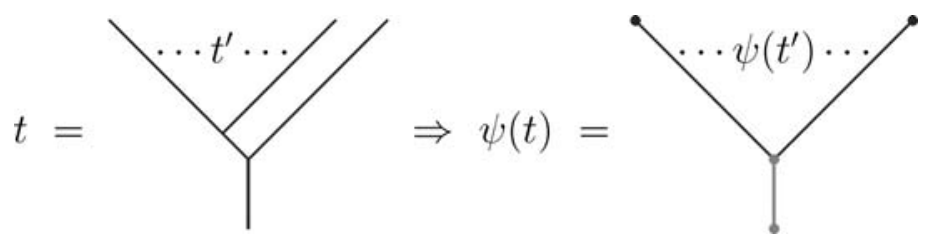


Finally, $\psi(\mid)=\bullet$ is the unique ordered tree with one node. For instance,

$$
\begin{aligned}
& \psi(Y)=\mathfrak{l}, \\
& \psi(Y)=\psi(Y) \backslash \psi(Y)=\bigvee \\
& \psi(Y)=\psi(Y) \backslash \psi(Y)=Y \backslash !=\bigvee .
\end{aligned}
$$

Note that $\psi$ identifies $\backslash$-irreducible planar binary trees with planted ordered trees.

In [3], we introduced a linear basis $M_{t}$ of $\mathcal{Y} S y m$, indexed by planar binary trees $t$, which is obtained from the original basis of Loday and Ronco by a process of Möbius inversion. We showed that $\mathcal{Y}$ Sym is a cofree graded coalgebra and the space $V$ of primitive elements is the linear span of the elements $M_{t}$ for $t$ a $\backslash$-irreducible planar binary trees [3, Theorem 7.1, Corollary 7.2]. The isomorphism $Q(V) \cong \mathcal{Y S Y m}$ is

$$
M_{t_{1}} \backslash \cdots \backslash M_{t_{k}} \longleftrightarrow M_{t_{1} \backslash \cdots \backslash t_{k}}
$$

The resulting grading by length on $\mathcal{Y} S y m$ is given by the number of $\backslash$-irreducible components in the decomposition of a planar binary tree $t$ (that is, the number of leaves that are directly attached to the right-most branch). The product of $\mathcal{Y}$ Sym does not preserve the grading by length. For instance,

$$
M_{Y} y \cdot M_{Y}=M_{Y} Y+M_{Y} Y+M_{Y Y}+2 \cdot M_{Y Y}+M_{Y} y .
$$

Consider the associated graded Hopf algebra, $\operatorname{gr}(\mathcal{Y} S y m)$. As coalgebras, $\operatorname{gr}(\mathcal{Y} S y m)=$ $\mathcal{Y}$ Sym but the product has been altered by removing terms of lower length (Section 1). Thus, in $\operatorname{gr}(\mathcal{Y} S y m)$,

$$
M_{Y} y \cdot M_{Y}=2 \cdot M_{Y Y}+M_{Y y}
$$

$\mathcal{Y}$ Sym admits a Hopf grading, given by the number of internal nodes of a planar binary tree (one less than the number of leaves). The isomorphism $\mathcal{Y} S y m \cong Q(V)$ matches this grading with the grading by weight. This also yields a grading on $\operatorname{gr}(\mathcal{Y} S y m)$, which corresponds to the grading by weight under the isomorphism $\operatorname{gr}(\mathcal{Y} S y m) \cong \operatorname{Sh}(V)$ of Proposition 1.5.

We relate the graded Hopf algebras $\operatorname{gr}(\mathcal{Y} S y m)$ and $\mathcal{H}_{O}$ (graded by one less than the number of leaves and one less than the number of nodes, respectively). The dual of $\operatorname{gr}(\mathcal{Y} S y m)$ is with respect to this grading. 
Theorem 2.5 There is an isomorphism of graded Hopf algebras $\Psi: \operatorname{gr}(\mathcal{Y} S y m)^{*} \rightarrow \mathcal{H}_{O}$ uniquely determined by

$$
M_{t}^{*} \mapsto \psi(t)
$$

for \-irreducible planar binary trees $t$.

Proof: According to the previous discussion, $\operatorname{gr}(\mathcal{Y} S y m)$ is the shuffle Hopf algebra on the subspace $V$ and the number of internal nodes corresponds to the grading by weight. Therefore $\operatorname{gr}(\mathcal{Y S y m})^{*}$ is the tensor Hopf algebra $T\left(V^{*}\right)$ on the graded dual space. Thus (2.6) determines a morphism of algebras $\Psi: \operatorname{gr}(\mathcal{Y} S y m)^{*} \rightarrow \mathcal{H}_{O}$. Since the number of nodes of $\psi(t)$ is the number of leaves of $t, \Psi$ preserves the Hopf gradings. Moreover, $\Psi$ preserves coproducts on a set of algebra generators of $\operatorname{gr}(\mathcal{Y} S y m)^{*}$ : the elements $M_{t}^{*}$ indexed by $\backslash$-irreducible planar binary trees are primitive generators of the tensor Hopf algebra, and their images $\psi(t)$ are primitive elements of $\mathcal{H}_{O}$ (since they are planted trees). Therefore, $\Psi$ is a morphism of Hopf algebras.

We complete the proof by showing that $\Psi$ is invertible.

Let $t$ be an arbitrary planar binary tree and $t=t_{1} \backslash t_{2} \backslash \cdots \backslash t_{k}$ the decomposition (2.3). Then $M_{t}^{*}=M_{t_{1}}^{*} \cdot M_{t_{2}}^{*} \cdots M_{t_{n}}^{*}$, and so

$$
\Psi\left(M_{t}^{*}\right)=\psi\left(t_{1}\right) \cdot \psi\left(t_{2}\right) \cdots \psi\left(t_{k}\right) .
$$

Since each $t_{i}$ is planted, Definition 2.2 shows that this product is the sum of all ordered trees obtained by attaching the root of $\psi\left(t_{k-1}\right)$ to a node of $\psi\left(t_{k}\right)$, and then attaching the root of $\psi\left(t_{k-2}\right)$ to a node of the resulting tree, and etc. The number of children of the root of such a tree is less than $k$, except when all the $\psi\left(t_{i}\right)$ are attached to the root, obtaining the ordered tree $\psi(t)=\psi\left(t_{1}\right) \backslash \psi\left(t_{2}\right) \backslash \cdots \backslash \psi\left(t_{k}\right)$.

Linearly ordering both ordered trees and planar binary trees so that trees with fewer components precede trees with more components (in the decompositions (2.1) and (2.3)), this calculation shows that

$$
\Psi\left(M_{t}^{*}\right)=\psi(t)+\text { trees of smaller order. }
$$

Thus $\Psi$ is bijective.

The main result of Grossman and Larson on the structure of $\mathcal{H}_{O}$ [10, Theorem 5.1] is contained in the proof of Theorem 2.5. We state it next.

Corollary 2.7 The set of planted ordered trees freely generates the algebra $\mathcal{H}_{O}$ of ordered trees. Moreover, $\mathcal{H}_{O}$ is isomorphic to the tensor Hopf algebra on the linear span of the set of planted trees.

Proof: As seen in the proof of Theorem $2.5, \mathcal{H}_{O} \cong T\left(V^{*}\right)$ as Hopf algebras. The isomorphism maps a basis of $V^{*}$ to the set of planted trees, so the result follows. 
Remark 2.8 We point out that one may construct an isomorphism of graded Hopf algebras $\operatorname{gr}(\mathcal{Y S y m})^{*} \cong \mathcal{H}_{O}$ from any bijection between the set of planted trees with $n$ nodes and the set of $\backslash$-irreducible planar binary trees with $n$ leaves, instead of the map $\psi$ we used. In fact, since they are tensor Hopf algebras, any degree-preserving bijection between the sets of generators determines a unique isomorphism of graded Hopf algebras.

The number of planted trees with $n+2$ nodes (or \-irreducible planar binary trees with $n+2$ leaves) is the Catalan number $\frac{1}{n+1}\left(\begin{array}{c}2 n \\ n\end{array}\right)$.

\section{The Hopf algebra of heap-ordered trees}

We show that the graded dual to $\operatorname{gr}(\mathfrak{S S y m})$ is isomorphic to the cocommutative Hopf algebra of heap-ordered trees defined by Grossman and Larson [10].

We first review the definition of the Hopf algebra of heap-ordered trees.

A heap-ordered tree is an ordered tree $x$ together with a labeling of the nodes (a bijection $\operatorname{Nod}(x) \rightarrow\{0,1, \ldots, n\})$ such that:

- The root of $x$ is labeled by 0 ;

- The labels increase as we move from a node to any of its children;

- The labels decrease as we move from left to right within the children of each node.

The heap-ordered trees with 1, 2, 3, and 4 nodes are shown below:

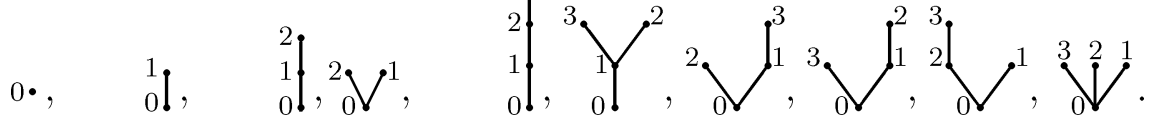

The constructions for ordered trees described in Section 2 may be adapted to the case of heap-ordered trees.

Let $x$ and $y$ be heap-ordered trees. Suppose $x$ has $k$ planted components (these are ordered trees). Given a function $f:[k] \rightarrow \operatorname{Nod}(y)$, the ordered tree $x \#_{f} y$ may be turned into a heap-ordered tree by keeping the labels of $y$ and incrementing the labels of $x$ uniformly by the highest label of $y$. Given a subset $S=\left\{i_{1}<\cdots<i_{p}\right\} \subseteq[k]$, the ordered tree $x_{S}$ may be turned into a heap-ordered tree by standardizing the labels, which is to replace the $i$ th smallest label by the number $i$, for each $i$.

Definition 3.1 The Grossman-Larson Hopf algebra $\mathcal{H}_{H O}$ of heap-ordered trees is the formal linear span of all heap-ordered trees with product and coproduct as follows. Given 
heap-ordered trees $x$ and $y$ as above, we set

$$
\begin{aligned}
& x \cdot y=\sum_{f:[k] \rightarrow \operatorname{Nod}(y)} x_{f} y, \\
& \Delta(x)=\sum_{S \subseteq[k]} x_{S} \otimes x_{S^{c}} .
\end{aligned}
$$

For instance,

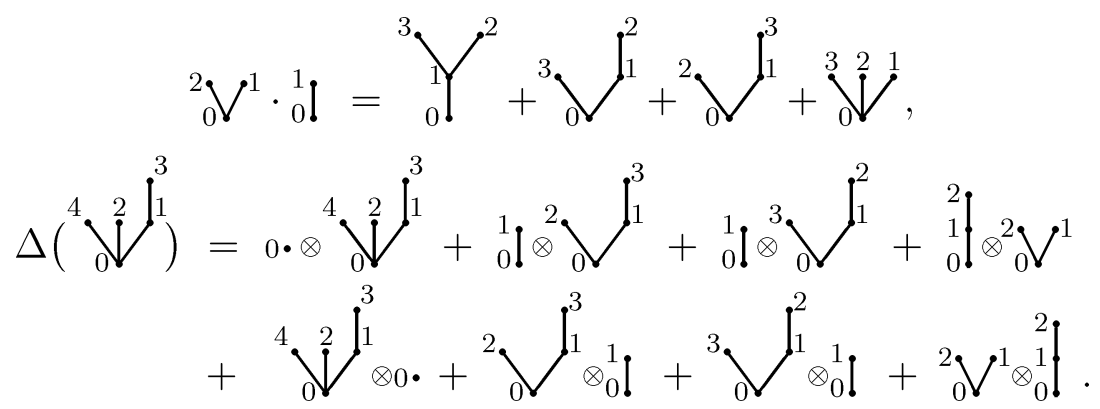

$\mathcal{H}_{H O}$ is a graded cocommutative Hopf algebra, where the degree of an ordered tree is one less than the number of nodes [10, Theorem 3.2].

Heap-ordered trees on $n+1$ nodes are in bijection with permutations on $n$ letters. We construct a permutation from such a tree by listing the labels of all non-root nodes in such way that the label of a node $i$ is listed to the left of the label of a node $j$ precisely when $i$ is below or to the left of $j$ (that is, when $i$ is a predecessor of $j$, or $i$ is among the left descendants of the nearest common predecessor between $i$ and $j$ ). For instance, the six heap-ordered trees on 4 nodes above correspond respectively to $123,132,213,312,231$, and 321 .

Let $\phi$ be the inverse bijection. Given a permutation $u$, the heap-ordered tree $\phi(u)$ is computed as follows. Let $u(1), \ldots, u(n)$ be the values of $u$ and set $u(0):=0$.

- Step 0. Start from a root labeled 0 .

- Step 1. Draw a child of the root labeled $u(1)$.

- Step $i, i=2, \ldots, n$. Draw a new node labeled $u(i)$. Let $j \in\{0, \ldots, i-1\}$ be the maximum index such that $u(i)>u(j)$. The new node is a child of the node drawn in step $j$, and it is placed to the right of any previous children of that node.

For instance,

$$
\phi(4231)=\sqrt[4]{2},
$$

and

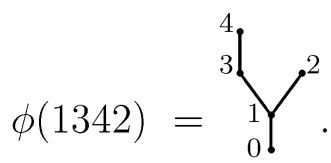


Given two heap-ordered trees $x$ and $y$, the ordered tree $x \backslash y$ may be turned into a heapordered tree by incrementing all labels of the nodes in $x$ by the maximum label of a node in $y$. For instance,

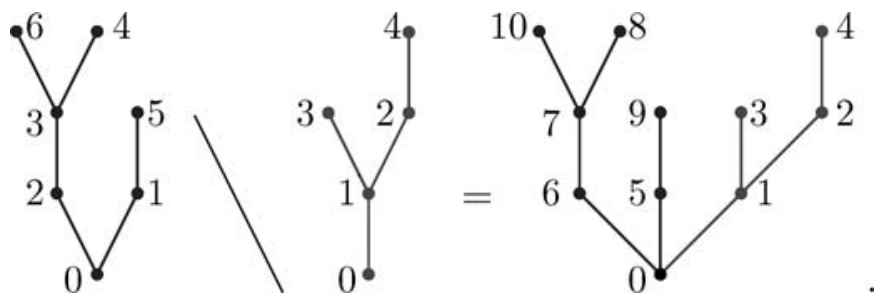

The operation $\backslash$ is associative on heap-ordered trees, so each such tree has a unique irreducible decomposition into \-irreducible ones. As for ordered trees, the heap-ordered trees that are planted are $\backslash$-irreducible. There are, however, many other $\backslash$-irreducible heapordered trees. For instance, while

$$
\lfloor!=\vee
$$

the heap-ordered tree

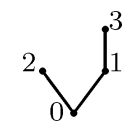

is $\backslash$-irreducible.

The operation $u \backslash v$ between permutations [19] is obtained by first listing the values of $u$, incremented by the highest value of $v$, and then listing the values of $v$ to its right. For instance,

$$
231 \backslash 21=45321
$$

A permutation $w$ has a global descent at position $p$ if $w=u \backslash v$ with $u$ a permutation of $p$ letters. Thus, the $\backslash$-irreducible permutations are the permutations with no global descents (see [2, Corollary 6.4] for their enumeration).

The definition of $\phi$ (or its inverse) makes it clear that

$$
\phi(u \backslash v)=\phi(u) \backslash \phi(v)
$$


for any permutations $u$ and $v$. In particular, \-irreducible heap-ordered trees correspond to $\backslash$-irreducible permutations under $\phi$.

In [2], we introduced a linear basis $M_{w}$ of $\mathfrak{S} S y m$, indexed by permutations $w$, which is obtained from the original basis of Malvenuto and Reutenauer by a process of Möbius inversion. We showed that $\mathfrak{S} S y m$ is a cofree graded coalgebra and the space $V$ of primitive elements is the linear span of the elements $M_{w}$ indexed by $\backslash$-irreducible permutations $w$ [2, Theorem 6.1, Corollary 6.3]. The isomorphism $Q(V) \cong \mathfrak{S S y m}$ is

$$
M_{w_{1}} \backslash \cdots \backslash M_{w_{k}} \leftrightarrow M_{w_{1} \backslash \cdots \backslash w_{k}} .
$$

The resulting grading by length on $\mathfrak{S} S y m$ is given by the number of $\backslash$-irreducible components in the decomposition of a permutation $w$. The product of $\mathfrak{S} S y m$ does not preserve this grading by length. For instance, in $\mathfrak{S} S y m$,

$$
M_{231} \cdot M_{1}=M_{2314}+M_{2413}+M_{2341}+2 \cdot M_{2431}+M_{3412}+2 \cdot M_{3421}+M_{4231} .
$$

In this product, $M_{231}$ has length $2, M_{1}$ has length 1 , and the only elements of length 3 are $M_{3421}$ and $M_{4231}$. Thus, in the associated graded Hopf algebra gr(SSym),

$$
M_{231} \cdot M_{1}=2 \cdot M_{3421}+M_{4231} .
$$

SSym admits a Hopf grading, in which a permutation on $n$ letters has degree $n$. The isomorphism $\mathfrak{S} S y m \cong Q(V)$ matches this grading with the grading by weight. This also yields a grading on $\operatorname{gr}(\mathfrak{S} S y m)$, which corresponds to the grading by weight under the isomorphism $\operatorname{gr}(\mathfrak{S} S y m) \cong \operatorname{Sh}(V)$ of Proposition 1.5.

Theorem 3.4 relates the dual of $\operatorname{gr}(\mathfrak{S} S y m)$ with respect to this grading, with $\mathcal{H}_{H O}$, graded by one less than the number of nodes.

We define the order of a heap-ordered tree $x$ to be the pair $(k, l)$, where $k$ is the number of planted components of $x$ and $l$ is the number of irreducible components of $x$. We use the following version of the lexicographic order to compare trees:

$$
(k, l)<(m, n) \text { if } \quad k<m \quad \text { or } \quad k=m \text { and } l>n .
$$

That is, trees with more planted components have higher order, but among trees with the same number of planted components, then those with fewer irreducible components have higher order.

Let $x$ be a heap-ordered tree and $\alpha$ an arbitrary element of $\mathcal{H}_{H O}$. The notation

$$
\alpha=x+\text { t.s.s.o. }
$$

indicates that $\alpha-x$ equals a linear combination of heap-ordered trees each of which is of strictly smaller order than $x$. Not every $\alpha$ can be written in this form, as several trees of the same order may appear in $\alpha$.

Lemma 3.2 If $\alpha=x+$ t.s.s.o. and $\beta=y+$ t.s.s.o., then $\alpha \cdot \beta=x \backslash y+$ t.s.s.o. 
Proof: Consider first the product of two heap-ordered trees $x^{\prime}$ and $y^{\prime}$ having orders $(k, l)$ and $(m, n)$ respectively. This is the sum of all heap-ordered trees obtained by attaching the planted components of $x^{\prime}$ to nodes of $y^{\prime}$. Every such tree will have fewer than $k+m$ planted components, except the tree obtained by attaching all planted components of $x^{\prime}$ to the root of $y^{\prime}$, which will be $x^{\prime} \backslash y^{\prime}$ and will have $k+m$ planted components.

Therefore, among the trees appearing in $\alpha \cdot \beta$, the ones with the maximum number of planted components are those of the form $x^{\prime} \backslash y^{\prime}$, with $x^{\prime}$ and $y^{\prime}$ having the same numbers of planted components as $x$ and $y$ respectively. Among these we find the tree $x \backslash y$. For any of the remaining trees with the maximum number of planted components, either $x^{\prime}$ has more irreducible components than $x$ or $y^{\prime}$ has more irreducible components than $y$, by hypothesis. Since the number of irreducible components of $x^{\prime} \backslash y^{\prime}$ is $l+n$, the tree $x^{\prime} \backslash y^{\prime}$ has more irreducible components than $x \backslash y$, and hence it is of smaller order.

Applying Lemma 3.2 inductively we deduce that any heap-ordered tree $x$ is the leading term in the product of its irreducible components. This implies that the set of irreducible heap-ordered trees freely generates the algebra $\mathcal{H}_{H O}$ of ordered trees. This result is due to Grossman and Larson [10, Theorem 6.3]. Irreducible heap-ordered trees are not necessarily primitive. We refine this result of Grossman and Larson, giving primitive generators and relating the structure of $\mathcal{H}_{\mathrm{HO}}$ explicitly to that of $\operatorname{gr}(\mathfrak{S} S y m)^{*}$.

We assume from now on that the base field $\mathbb{k}$ is of characteristic 0 .

We need one more tool: the first Eulerian idempotent [9], [17, Section 4.5.2], [27, Section 8.4]. For any graded connected Hopf algebra $H$, the identity map id : $H \rightarrow H$ is locally unipotent with respect to the convolution product of $\operatorname{End}(H)$. Here 1 denotes the composite $H \stackrel{\epsilon}{\rightarrow} \mathbb{k} \stackrel{u}{\rightarrow} H$ of the counit and unit maps of $H$ (the unit element for the convolution product). Therefore,

$$
\mathbf{e}:=\log (\mathrm{id})=\sum_{n \geq 1} \frac{(-1)^{n+1}}{n}(\mathrm{id}-1)^{* n}
$$

is a well-defined linear endomorphism of $H$. The crucial fact is that if $H$ is cocommutative, this operator is a projection onto the space of primitive elements of $H: \mathbf{e}: H \rightarrow$ $P(H)$ [25], [28, pp. 314-318].

Lemma 3.3 Let $x$ be a \-irreducible heap-ordered tree. Then

$$
\mathbf{e}(x)=x+\text { t.s.s.o. }
$$

Proof: In any graded connected Hopf algebra $H$, the map id -1 is the projection of $H$ onto the part of positive degree, and the convolution power $(\mathrm{id}-1)^{* n}$ equals the map $m^{(n-1)} \circ(\mathrm{id}-1)^{\otimes n} \circ \Delta^{(n-1)}$. Let $x$ be a heap-ordered tree with $k$ planted components. 
Iterating the coproduct of $\mathcal{H}_{H O}$ (Definition 3.1) gives

$$
\Delta^{(n-1)}(x)=\sum_{S_{1} \sqcup \cdots \sqcup S_{n}=[k]} x_{S_{1}} \otimes \cdots \otimes x_{S_{n}},
$$

the sum over all ordered decompositions of $[k]$ into $n$ disjoint subsets. Applying (id -1$)^{\otimes n}$ to this sum has the effect of erasing all terms corresponding to decompositions involving at least one empty set. Therefore,

$$
(\mathrm{id}-1)^{* n}(x)=\sum_{\substack{S_{1} \sqcup \cdots \sqcup S_{n}=[k] \\ S_{i} \neq \varnothing}} x_{S_{1}} \cdots x_{S_{n}},
$$

the sum now over all set-compositions of $[k]$ (ordered decompositions into non-empty disjoint subsets). In particular, this sum is 0 when $n>k$. Thus,

$$
\mathbf{e}(x)=\sum_{n=1}^{k} \frac{(-1)^{n+1}}{n} \sum_{\substack{S_{1} \sqcup \cdots \sqcup S_{n}=[k] \\ S_{i} \neq \emptyset}} x_{S_{1}} \cdots x_{S_{n}} .
$$

By Lemma 3.2, $x_{S_{1}} \cdots x_{S_{n}}=x_{S_{1}} \backslash \cdots \backslash x_{S_{n}}+$ t.s.s.o. Each tree $x_{S_{1}} \backslash \cdots \backslash x_{S_{n}}$ has $k$ planted components (as many as $x$ ) and at least $n$ irreducible components. Hence, among these trees, the one of highest order is $x$, which corresponds to the trivial decomposition of $[k]$ into $n=1$ subset. Thus, among all trees appearing in $\mathbf{e}(x)$, there is one of highest order and it is $x$.

For example, if

$$
x=2 \int^{3}
$$

then

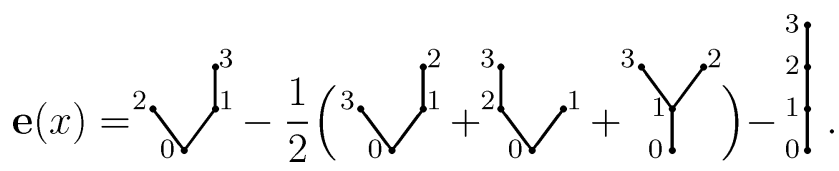

The tree $x$ is of order $(2,1)$, the next two trees are of order $(2,2)$, and the last two of order $(1,1)$.

Theorem 3.4 Assume char $(\mathbb{k})=0$. There is an isomorphism of graded Hopf algebras $\Phi: \operatorname{gr}\left(\mathrm{SSym}^{*} \rightarrow \mathcal{H}_{\mathrm{HO}}\right.$ uniquely determined by

$$
M_{w}^{*} \mapsto \mathbf{e}(\phi(w))
$$

for $w$ a \-irreducible permutation. 
Proof: By the discussion preceding Lemma 3.2, $\operatorname{gr}(\mathfrak{S S y m})^{*} \cong T\left(V^{*}\right)$. Therefore, (3.5) determines a morphism of graded algebras $\Phi$. Since $\mathcal{H}_{H O}$ is cocommutative, $\mathbf{e}(\phi(w))$ is a primitive element of $\mathcal{H}_{\mathrm{HO}}$. Thus $\Phi$ preserves primitive elements and hence it is a morphism of Hopf algebras.

It remains to verify that $\Phi$ is invertible. Let $w=w_{1} \backslash \cdots \backslash w_{k}$ be the irreducible decomposition of a permutation $w$. Let $x:=\phi(w)$. Since $\phi$ preserves the operations $\backslash$, the irreducible components of $x$ are $x_{i}:=\phi\left(w_{i}\right), i=1, \ldots, k$. On the other hand, $M_{w}^{*}=M_{w_{1}}^{*} \cdots M_{w_{k}}^{*}$, so

$$
\Phi\left(M_{w}^{*}\right)=\mathbf{e}\left(x_{1}\right) \cdots \mathbf{e}\left(x_{k}\right)
$$

From Lemmas 3.2 and 3.3 we deduce

$$
\Phi\left(M_{w}^{*}\right)=x_{1} \backslash \cdots \backslash x_{k}+\text { t.s.s.o. }=\phi(w)+\text { t.s.s.o. }
$$

As in the proof of Theorem 2.5 , this shows that $\Phi$ is invertible, by triangularity.

Let $W$ be the graded space where $W_{n}$ is spanned by the elements $M_{w}^{*}$, for $w$ an irreducible permutation of $[n]$. From the proof of Theorem 3.4, we deduce the following corollary.

Corollary 3.6 The Hopf algebra $\mathcal{H}_{H}$ of ordered trees is isomorphic to the tensor Hopf algebra on a graded space $W=\oplus_{n \geq 0} W_{n}$ with $\operatorname{dim} W_{n}$ equal to the number of irreducible heap-ordered trees on $n+1$ nodes (or the number of irreducible permutations of $[n]$ ).

Remark 3.7 As pointed out to us by Holtkamp, the use of the Eulerian idempotent in Theorem 3.4 is similar to that encountered in a proof of the Milnor-Moore theorem [23, Theorem 5.18], [26, Theorem 4.5].

\section{Acknowledgments}

We thank Loïc Foissy and Ralf Holtkamp for interesting comments and remarks.

\section{References}

1. Marcelo Aguiar, Nantel Bergeron, and Frank Sottile, "Combinatorial Hopf algebras and generalized DehnSommerville relations," math. CO/0310016. To appear in Compos. Math.

2. Marcelo Aguiar and Frank Sottile, "Structure of the Malvenuto-Reutenauer Hopf algebra of permutations," Adv. Math. 191(2) (2005), 225-275.

3. Marcelo Aguiar and Frank Sottile, "Structure of the Hopf algebra of planar binary trees of Loday and Ronco," math. CO/ 0409022 , 2004. To appear in Journal of Algebra.

4. Frédéric Chapoton and Muriel Livernet, "Pre-Lie algebras and the rooted trees operad," Internat. Math. Res. Notices (8) (2001), 395-408.

5. A. Connes and D. Kreimer, "Hopf algebras, renormalization and noncommutative geometry," Comm. Math. Phys. 199 (1998), 203-242.

6. L. Foissy, "Les algèbres de Hopf des arbres enracinés décorés, I", Bull. Sci. Math. 126(3) (2002), 193-239.

7. L. Foissy, "Les algèbres de Hopf des arbres enracinés décorés, II," Bull. Sci. Math. 126(4) (2002), $249-288$. 
8. Israel M. Gelfand, Daniel Krob, Alain Lascoux, Bernard Leclerc, Vladimir S. Retakh, and Jean-Yves Thibon, "Noncommutative symmetric functions," Adv. Math. 112(2) (1995), 218-348.

9. Murray Gerstenhaber and S.D. Schack, "The shuffle bialgebra and the cohomology of commutative algebras," J. Pure Appl. Algebra 70(3) (1991), 263-272.

10. Robert Grossman and Richard G. Larson, "Hopf-algebraic structure of families of trees," J. Algebra 126(1) (1989), 184-210.

11. Robert Grossman and Richard G. Larson, "Solving nonlinear equations from higher order derivations in linear stages," Adv. Math. 82(2) (1990), 180-202.

12. Michiel Hazewinkel, "Generalized overlapping shuffle algebras," Pontryagin Conference, 8, Algebra (Moscow, 1998). J. Math. Sci. (New York) 106(4) (2001), 3168-3186.

13. Michael E. Hoffman, "Quasi-shuffle products," J. Algebraic Combin. 11(1) (2000), 49-68.

14. Michael E. Hoffman, "Combinatorics of rooted trees and Hopf algebras," Trans. Amer. Math. Soc. 355 (2003), 3795-3811.

15. Ralf Holtkamp, "Comparison of Hopf algebras on trees," Arch. Math. (Basel) 80(4) (2003), 368-383.

16. Samuel K. Hsiao, "Structure of the peak Hopf algebra of quasi-symmetric functions," 2002.

17. Jean-Louis Loday, Cyclic homology, Grundlehren der Mathematischen Wissenschaften, 301. Springer-Verlag, Berlin, 1998. xx+513 pp

18. Jean-Louis Loday and María O. Ronco, "Hopf algebra of the planar binary trees," Adv. Math. 139(2) (1998), 293-309.

19. Jean-Louis Loday and María O. Ronco, "Order structure on the algebra of permutations and of planar binary trees," J. Algebraic Combin. 15 (2002), 253-270.

20. Jean-Louis Loday and María O. Ronco, "On the structure of cofree Hopf algebras," math. QA/0405330, 2004.

21. Claudia Malvenuto, "Produits et coproduits des fonctions quasi-symétriques et de l'algèbre des descents," no. 16, Laboratoire de combinatoire et d'informatique mathématique (LACIM), Univ. du Québec à Montréal, Montréal, 1994.

22. Claudia Malvenuto and Christophe Reutenauer, "Duality between quasi-symmetric functions and the Solomon descent algebra," J. Algebra 177(3) (1995), 967-982.

23. John W. Milnor and John C. Moore, "On the structure of Hopf algebras," Ann. of Math. 81(2) (1965), 211-264

24. Susan Montgomery, "Hopf algebras and their actions on rings," CBMS Regional Conference Series in Mathematics 82, American Mathematical Society, Providence, RI, 1993.

25. Frédéric Patras, "L'algèbre des descentes d'une bigèbre graduée," J. Algebra 170(2) (1994), 547-566.

26. Daniel Quillen, "Rational homotopy theory," Ann. of Math. 90(2) (1969), 205-295.

27. Christophe Reutenauer, Free lie algebras, The Clarendon Press Oxford University Press, New York, 1993, Oxford Science Publications.

28. William R. Schmitt, “Incidence Hopf algebras,” J. Pure Appl. Algebra 96(3) (1994), 299-330

29. Manfred Schocker, "The peak algebra of the symmetric group revisited," 2002. math. RA/ 0209376

30. Richard P. Stanley, Enumerative combinatorics. Vol. 1, Cambridge University Press, Cambridge, 1997, With a foreword by Gian-Carlo Rota, Corrected reprint of the 1986 original.

31. Richard P. Stanley, Enumerative combinatorics. Vol. 2, Cambridge University Press, Cambridge, 1999, With a foreword by Gian-Carlo Rota and appendix 1 by Sergey Fomin.

32. John R. Stembridge, "Enriched P-partitions," Trans. Amer. Math. Soc. 349(2) (1997), 763-788.

33. Moss E. Sweedler, Hopf algebras, Mathematics Lecture Note Series W. A. Benjamin, Inc., New York, 1969 vii+336 pp. 